\title{
Hidradenitis suppurativa geht aufs Herz
}

\section{Hier steht eine Anzeige.}

\section{Patienten mit Hidradenitis suppurativa/Acne inversa haben ein erhöhtes Risiko für kardiovaskuläre Komplikationen. Dänische Ärzte haben dieses Risiko jetzt in Zahlen gefasst.}

$D$ änische Ärzte haben den Zusammenhang von kardiovaskulären Ereignissen bei Patienten mit Hidradenitis suppurativa/Acne inversa (HS/AI) anhand einer Fall-Kontroll-Studie mit 5.964 HS/AI-Patienten analysiert. Den Patienten - mittleres Alter 37 Jahre, 73 \% Frauen - waren im Verhältnis 1:5 Kontrollpersonen aus der dänischen Bevölkerung ohne HS/AI $(n=29.404)$ gegenübergestellt worden. Keiner der Teilnehmer hatte einen Myokardinfarkt oder einen Schlaganfall in der Anamnese.

Während der mittleren Nachbeobachtungszeit von sieben Jahren wurden in der Gesamtgruppe 200 Herzinfarkte, 282 ischämische Schlaganfälle und 800 Todesfälle, davon 183 mit kardiovaskulärer Ursache, dokumentiert. Jedes dieser Ereignisse trat in der HS/AI-Gruppe signifikant häufiger auf als in der Referenzgruppe. Auch wenn andere Einflussfaktoren wie Alter, Geschlecht, Rauchverhalten, Begleiterkrankungen, Medikation und sozioökonomischer Status berücksichtigt wurden, waren in der HS/AI-Gruppe signifikante Steigerungen der Herzinfarkte um $57 \%$, der Schlaganfälle um $33 \%$, der kardiovaskulären Todesfälle um $95 \%$ und der Todesfälle überhaupt um $35 \%$ zu verzeichnen.

Der zusätzliche Vergleich der HS/AI-Patienten mit 13.093 Patienten mit schwerer Psoriasis (definiert durch eine systemische Psoriasistherapie) ergab vergleichbare Inzidenzraten für Herzinfarkt, Apoplex und Tod. Bei der kardiovaskulären Mortalität schnitten allerdings die HS/AI-Patienten nochmals schlechter ab, mit einer um 58 \% höheren Inzidenz. Die kardiovaskuläre Gefährdung von Psoriasispatienten ist bekannt; sie ist ähnlich hoch wie bei Diabetespatienten.

Fazit: Herzinfarkt, Schlaganfall und kardiovaskulärer Tod treten bei Patienten mit HS/AI nicht nur deutlich häufiger auf als in der Allgemeinbevölkerung. Die Ereignisraten sind auch mindestens so hoch wie bei Patienten mit schwerer Psoriasis. Die Autoren gehen davon aus, dass die Entzündung bei HS/AI bei der Entstehung der atherosklerotischen Komplikationen eine wichtige Rolle spielt. Die inflammatorischen Prozesse sind nicht lokal beschränkt, sondern lassen sich auch systemisch nachweisen, etwa durch erhöhte Blutspiegel von Tumornekrosefaktor, Leukozyten und CRP. Die Autoren weisen außerdem darauf hin, dass viele HS/AI-Patienten aufgrund von etablierten Risikofaktoren wie Rauchen, Adipositas, metabolischem Syndrom und Diabetes Kandidaten für Herz-Kreislauf-Erkrankungen sind.

Dr. Beate Schumacher

Egeberg A et al. Risk of Major Adverse Cardiovascular Events and All-Cause Mortality in Patients With Hidradenitis Suppurativa. JAMA Dermatol 2016; 152: $429-34$ 